\title{
Gewaltkriminalität - auf den Boden kriminologischer Erkenntnisse gestellt
}

\author{
zu Michael Walter: Gewaltkriminalität, 1. Auflage, 2006
}

Heribert Ostendorf

Die Gewaltkriminalität beherrscht die kriminalpolitische Diskussion. Medienmacher und Politiker bedienen sich dieser Kriminalitätsform. Der Bürger entwickelt Ängste in der Annahme einer dramatischen Steigerung: „Die innere Sicherheit ist bedroht. “ Infolgedessen wird der Ruf nach härteren Strafen, der Ruf nach wirksamer Abschreckung potentieller Täter und Sicherung der Gesellschaft überführter Täter bis hin zum dauerhaften Wegsperren lauter. Die „Heizspirale“ von öffentlicher Gewaltdramatisierung, Bürgerängsten und Forderungen nach Strafverschärfungen dreht sich immer schneller (Scherer: „Politisch-publizistischer Verstärkerkreislauf“). Mit dem „Büchlein“ (80 Textseiten) „Gewaltkriminalität“ legt Michael Walter, Direktor des Instituts für Kriminologie an der Universität Köln, eine Analyse dieser „gesellschaftlich konstituierten Kriminalität" (Vorwort) vor und wirbt für einen vernünftigen Umgang mit Gewaltkriminalität. Der Untertitel lautet dementsprechend: Erscheinungsformen, Ursachen, Antworten. Walter kann hierbei auf umfassendere Arbeiten, wie insbesondere auf das Standardwerk „Jugendkriminalität“ (3. Auflage) zurückgreifen.

Im ersten Kapitel führt Walter in „die Kriminalität“ ein, die es so nicht gibt, die höchst unterschiedlich ist. Es folgt eine kriminologische Deliktseinteilung, wobei - holzschnittartig - zwischen täterbezogenen, tatbezogenen und opferbezogenen Gesichtspunkten unterschieden wird. Mit den täterbezogenen Gesichtspunkten werden Tätertypen vorgestellt, wobei Walter diese selbst wieder auflöst, auf zusätzliche tatbezogene Gesichtspunkte, auf gesellschaftliche Prozesse, z. B. der Selektierung bei der „Ausländerkriminalität“ hinweist. Es geht hier zunächst nur um die Erscheinungsformen von Kriminalität, noch nicht um Erklärungsansätze. In diesem Kontext wird dann Gewaltkriminalität verortet, an der unterschiedlichste „Tätertypen " beteiligt sind und die gleichsam die Urform der Kriminalität darstellt.

Das zweite Kapitel ist überschrieben mit „Kriminologische Erfassung von Gewaltphänomenen“. Zunächst wird die gesellschaftliche Wahrnehmung von Gewalt und Gewaltkriminalität analysiert. Walter stellt im Allgemeinen eine wachsende Sensibilisierung gegenüber Gewaltphänomenen fest, die einerseits im Anschluss an Norbert Elias als zivilisatorische Leistung anerkannt wird, die andererseits aber auch zu Dramatisierungen führen kann. Umgekehrt stellt Walter aber auch eine Normalisierung fest: „Gewalt ist Teil der Kultur“ (S. 30) - besser gesagt der Unkultur. So werden eine Lust an der Gewalt, eine strukturelle Gewalt, eine staatliche Gegengewalt sowie
Eigengewalt (in Form von Kriegsführungen), häuslich-intime Gewalt und „Ehrengewalt“ angesprochen. Weiter unterscheidet Walter zwischen Gewalt in Gedanken und in der realen Ausführung. In diesem Zusammenhang wird die mediale Beeinflussung zur Gewalt diskutiert. Nach Walter liegt eine Stimulationswirkung nahe. Ein anderes Ergebnis lautet: Die medial vermittelte Gewaltkriminalität entspricht nicht der Wirklichkeit.

Nach dieser phänomenologischen Beschreibung folgt eine definitorische Klärung der Gewaltbegriffe sowie die polizeistatistische Erfassung der Gewaltkriminalität: 3 \% der Gesamtkriminalität. Es folgen die Erklärungsansätze. Hier werden auch die im Rahmen der zunächst erfolgten Deliktseinteilung nur am Rande gestreiften gesellschaftlichen Strukturen behandelt. Besonderes Augenmerk richtet der Autor im Weiteren auf die kultur-historischen und zwischenstaatlichen Unterschiede in den Gewaltbewertungen sowie auf die Selektierung der Gewaltkriminalität im Hellfeld: Vieles bleibt verborgen trotz gestiegener Anzeigebereitschaft.

Im dritten Kapitel wendet sich der Autor der Frage zu, wie strafjustiziell sowie gesellschaftlich mit Gewaltkriminalität umgegangen werden sollte. Walter setzt wie viele andere auf die Prävention, warnt aber wie nur wenige vor strukturellen Gefahren einer umfassenden Prävention. Auch steht nach Walter eine Erfolgsmessung der vielfachen Präventionsaktivitäten erst am Anfang. Er vermisst eine Gewaltprävention zugunsten älterer Menschen.

Michael Walter hat ein sehr gut lesbares - mit Beispielen aus der Weltliteratur veranschaulicht - , ein höchst informatives, in sich geschlossenes Werk zur Gewaltkriminalität vorgelegt, das sowohl für die Aus- und Weiterbildung von Berufsgruppen, die mit Gewaltkriminalität zu tun haben, als auch zur Bürgeraufklärung in besonderer Weise empfehlenswert ist. Es ist darüber hinaus auch für „Fachleute“ anregend und nachdenklich stimmend. Wer sich auf ein „Aufklärungsbuch “ einlässt und damit eigene sowie fremde Forschungsergebnisse in allgemein-verständlicher Sprache wiedergibt, setzt sich leicht dem Vorwurf der Oberflächlichkeit, der nicht genügenden Differenzierung aus. Hierbei ist es umgekehrt eine Kunst, das Fachspezifische „zu übersetzen“ und das Wesentliche herauszuarbeiten. Ein kluges Buch, das zu kriminalpolitischer Klugheit rät.

Leiter der Forschungsstelle für Jugendstrafrecht und Kriminalprävention an der Universität Kiel

\section{Vorschau:}

Heft 3/2008 erscheint im September 2008

Thema:

40 Jahre Strafrechtsreform 